\title{
The Bohr phenomenon for analytic functions on shifted disks
}

\section{Molla Basir Ahamed, Vasudevarao Allu and Himadri Halder}

\begin{abstract}
In this paper, we investigate the Bohr phenomenon for the class of analytic functions defined on the simply connected domain

$$
\Omega_{\gamma}=\left\{z \in \mathbb{C}:\left|z+\frac{\gamma}{1-\gamma}\right|<\frac{1}{1-\gamma}\right\} \text { for } 0 \leq \gamma<1 .
$$

We study improved Bohr radius, Bohr-Rogosinski radius and refined Bohr radius for the class of analytic functions defined in $\Omega_{\gamma}$, and obtain several sharp results.

\section{Bohrin ilmiö analyyttisille funktioille siirretyissä kiekoissa}

Tiivistelmä. Tarkastelemme Bohrin ilmiötä yhdesti yhtenäisessä alueessa

$$
\Omega_{\gamma}=\left\{z \in \mathbb{C}:\left|z+\frac{\gamma}{1-\gamma}\right|<\frac{1}{1-\gamma}\right\}
$$

määriteltyjen analyyttisten funktioiden luokassa, missä $0 \leq \gamma<1$. Tutkimme parannettua Bohrin sädettä, Bohrin-Rogosinskin sädettä sekä tarkennettua Bohrin sädettä alueessa $\Omega_{\gamma}$ määriteltyjen analyyttisten funktioiden luokassa ja saamme useita tarkkoja tuloksia.
\end{abstract}

\section{Introduction and preliminaries}

Let $\mathcal{B}(\mathbb{D})$ be the class of analytic functions in unit disk $\mathbb{D}=\{z \in \mathbb{C}:|z|<1\}$ such that $f(\mathbb{D}) \subseteq \overline{\mathbb{D}}$. The classical Bohr theorem for functions $f \in \mathcal{B}(\mathbb{D})$ says that if $f(z)=\sum_{n=0}^{\infty} a_{n} z^{n}$, then its associated majorant series $M_{f}(r)$ satisfies the following inequality

$$
M_{f}(r):=\sum_{n=0}^{\infty}\left|a_{n}\right| r^{n} \leq 1 \quad \text { for } \quad|z|=r \leq \frac{1}{3}
$$

and the constant $1 / 3$, called Bohr radius for the class $\mathcal{B}(\mathbb{D})$, cannot be improved. The inequality (1.1) is known as classical Bohr inequality (1.1) for the class $\mathcal{B}(\mathbb{D})$. The Bohr inequality was first obtained by Harald Bohr [24] in 1914 with the constant $1 / 6$. The optimal value $1 / 3$, which is called the Bohr radius for disk case was later established independently by Weiner, Riesz and Schur. For the proofs we refer to [41] and [42]. The notion of Bohr inequality has been generalized to several complex variables by finding the multidimensional Bohr radius. We refer the reader to the articles $[6,8,23,37]$. For more information and intriguing aspects on Bohr phenomenon, we suggest the reader to glance through the articles [1]-[5], [7]-[13] and [16]-[18]. Bohr phenomenon for operator valued functions have been extensively studied by Bhowmik and Das (see [21, 22]).

https://doi.org/10.54330/afm.112561

2020 Mathematics Subject Classification: Primary 30C45, 30C50, 30C80.

Key words: Simply connected domain, bounded analytic functions, improved Bohr radius, Bohr-Rogosinski radius, refined Bohr radius and Bohr inequality.

(c) 2022 The Finnish Mathematical Society 
The main aim of this article is to study the Bohr inequality for the class of analytic functions that are defined in a general simply connected domain in the complex plain. Let $\Omega$ be a simply connected domain containing $\mathbb{D}$ and $\mathcal{B}(\Omega)$ be the class of analytic functions in $\Omega$ such that $f(\Omega) \subseteq \overline{\mathbb{D}}$. We define the Bohr radius $B=B_{\Omega}$ for the class $\mathcal{B}(\Omega)$ by

$$
B:=\sup \left\{r \in(0,1): \sum_{n=0}^{\infty}\left|a_{n}\right| r^{n} \leq 1 \text { for all } f \in \mathcal{B}(\Omega) \text { with } f(z)=\sum_{n=0}^{\infty} a_{n} z^{n}, z \in \mathbb{D}\right\} .
$$

In particular, if $\Omega=\mathbb{D}$, then $B_{\mathbb{D}}=1 / 3$, which is the classical Bohr radius for the class $\mathcal{B}(\mathbb{D})$. Let $\mathbb{D}(a, r):=\{z \in \mathbb{C}:|z-a|<r\}$. Clearly, $\mathbb{D}:=\mathbb{D}(0,1)$. Let $0 \leq \gamma<1$. We consider the disk $\Omega_{\gamma}$ defined by

$$
\Omega_{\gamma}:=\left\{z \in \mathbb{C}:\left|z+\frac{\gamma}{1-\gamma}\right|<\frac{1}{1-\gamma}\right\} .
$$

It is easy to see that $\Omega_{\gamma}$ always contains the unit disk $\mathbb{D}$. In 2010 , the notion of classical Bohr inequality (1.1) has been generalized by Fournier and Ruscheweyh [28] to the class $\mathcal{B}\left(\Omega_{\gamma}\right)$. More precisely,

Theorem 1.2. [28] For $0 \leq \gamma<1$, let $f \in \mathcal{B}\left(\Omega_{\gamma}\right)$, with $f(z)=\sum_{n=0}^{\infty} a_{n} z^{n}$ in $\mathbb{D}$. Then,

$$
\sum_{n=0}^{\infty}\left|a_{n}\right| r^{n} \leq 1 \quad \text { for } r \leq \rho:=\frac{1+\gamma}{3+\gamma}
$$

Moreover, $\sum_{n=0}^{\infty}\left|a_{n}\right| \rho^{n}=1$ holds for a function $f(z)=\sum_{n=0}^{\infty} a_{n} z^{n}$ in $\mathcal{B}\left(\Omega_{\gamma}\right)$ if, and only if, $f(z)=c$ with $|c|=1$.

In this article, we study the Bohr-Rogosinski radius for the class $\mathcal{B}\left(\Omega_{\gamma}\right)$. In 2017, Kayumov and Ponnusamy [34] introduced Bohr-Rogosinski radius motivated from Rogosinski radius for bounded analytic functions in $\mathbb{D}$. Rogosinski radius is defined as follows: Let $f(z)=\sum_{n=0}^{\infty} a_{n} z^{n}$ be analytic in $\mathbb{D}$ and its corresponding partial sum of $f$ is defined by $S_{N}(z):=\sum_{n=0}^{N-1} a_{n} z^{n}$. Then, for every $N \geq 1$, we have $\left|\sum_{n=0}^{N-1} a_{n} z^{n}\right|<1$ in the disk $|z|<1 / 2$ and the radius $1 / 2$ is sharp. Motivated by Rogosinski radius, Kayumov and Ponnusamy have considered the Bohr-Rogosinski $\operatorname{sum} R_{N}^{f}(z)$ is defined by

$$
R_{N}^{f}(z):=|f(z)|+\sum_{n=N}^{\infty}\left|a_{n}\right||z|^{n}
$$

It is worth to point out that $\left|S_{N}(z)\right|=\left|f(z)-\sum_{n=N}^{\infty} a_{n} z^{n}\right| \leq\left|R_{N}^{f}(z)\right|$. Thus, it is easy to see that the validity of Bohr-type radius for $R_{N}^{f}(z)$, which is related to the classical Bohr sum (Majorant series) in which $f(0)$ is replaced by $f(z)$, gives Rogosinski radius in the case of bounded analytic functions in $\mathbb{D}$. There has been significant and extensive research carried out on Improved-Bohr inequality and BohrRogosinski radius (see [14, 29, 30, 34, 31, 32, 33, 35, 36, 38]).

Lemma 1.4. [40] Let $a \in \mathbb{D}$ and $f \in \mathcal{B}(\mathbb{D})$ with

$$
f(z)=\sum_{n=0}^{\infty} \alpha_{n}(z-a)^{n}, \quad|z-a| \leq 1-|a| .
$$


Then,

$$
\left|\alpha_{n}\right| \leq(1+|a|)^{n-1} \frac{1-\left|\alpha_{0}\right|^{2}}{\left(1-|a|^{2}\right)^{n}}, n \geq 1
$$

Recently, Evdoridis et al. [27] obtained the following coefficient bounds for functions defined in $\Omega_{\gamma}$.

Lemma 1.5. [27] For $\gamma \in[0,1)$, let

$$
\Omega_{\gamma}=\left\{z \in \mathbb{C}:\left|z+\frac{\gamma}{1-\gamma}\right|<\frac{1}{1-\gamma}\right\}
$$

and let $f$ be an analytic function in $\Omega_{\gamma}$, bounded by 1 , with the series representation $f(z)=\sum_{n=0}^{\infty} a_{n} z^{n}$ in the unit disk $\mathbb{D}$. Then

$$
\left|a_{n}\right| \leq \frac{1-\left|a_{0}\right|^{2}}{1+\gamma} \quad \text { for } n \geq 1 \text {. }
$$

\section{Main results}

Before we state an improved version of inequality of Theorem 1.2, we prove the following lemma.

Lemma 2.1. Let $g: \mathbb{D} \rightarrow \overline{\mathbb{D}}$ be an analytic function, $m(\geq 2)$ be an integer, and let $\gamma \in \mathbb{D}$ be such that $g(z)=\sum_{n=0}^{\infty} \alpha_{n}(z-\gamma)^{n}$ for $|z-\gamma| \leq 1-|\gamma|$. Then

$$
\left|\alpha_{0}\right|+\sum_{n=1}^{\infty}\left(\left|\alpha_{n}\right|+\beta\left|\alpha_{n}\right|^{m}\right) \rho^{n} \leq 1 \quad \text { for } \rho \leq \rho_{0}:=\left(1-\gamma^{2}\right) /(3+\gamma),
$$

where

$$
\beta=\frac{(1-\gamma)^{m}(3+\gamma)-\left(1-\gamma^{2}\right)}{8(m-1)} \quad \text { for } 0 \leq \gamma \leq \gamma_{*}<1,
$$

where $\gamma_{*}$ is the smallest root of the equation $(1-\gamma)^{m}(3+\gamma)+\gamma^{2}-1=0$.

Using Lemma 2.1, we obtain the following improved version of Theorem 1.2 for the class $\mathcal{B}\left(\Omega_{\gamma}\right)$.

Theorem 2.3. For $0 \leq \gamma<1$, and integer $m(\geq 2)$, let $f \in \mathcal{B}\left(\Omega_{\gamma}\right)$ with $f(z)=\sum_{n=0}^{\infty} a_{n} z^{n}$ for $z \in \mathbb{D}$, then we have

$$
\left|a_{0}\right|+\sum_{n=1}^{\infty}\left(\left|a_{n}\right|+\beta \frac{\left|a_{n}\right|^{m}}{(1-\gamma)^{(m-1) n}}\right) r^{n} \leq 1 \quad \text { for } r \leq r_{0}=\frac{1+\gamma}{3+\gamma},
$$

where $\beta$ as in Lemma 2.1. Furthermore, the quantities $\beta$ and $(1+\gamma) /(3+\gamma)$ cannot be improved.

Figure 1 demonstrates values of $\gamma_{*}$ in $[0,1)$ for which $\beta(\gamma)>0$ with $0 \leq \gamma \leq$ $\gamma_{*}<1$. The values of $\gamma_{*}$ are $\gamma_{*}(10)=0.1083, \gamma_{*}(21)=0.0519, \gamma_{*}(50)=0.0219$ and $\gamma_{*}(100)=0.011$.

Lemma 2.4. Let $g: \mathbb{D} \rightarrow \overline{\mathbb{D}}$ be an analytic function, $\lambda \in[0,512 / 243]$ and let $\gamma \in \mathbb{D}$ be such that $g(z)=\sum_{n=0}^{\infty} \alpha_{n}(z-\gamma)^{n}$ for $|z-\gamma|<1-|\gamma|$. Then

$$
\sum_{n=0}^{\infty}\left|\alpha_{n}\right| \rho^{n}+\left(\frac{8}{9}-\frac{27}{64} \lambda\right)\left(\frac{S_{\rho}^{\gamma}}{\pi}\right)+\lambda\left(\frac{S_{\rho}^{\gamma}}{\pi}\right)^{2} \leq 1 \quad \text { for } \rho \leq \rho_{0}=\frac{1-|\gamma|^{2}}{3+|\gamma|},
$$

where $S_{\rho}^{\gamma}$ denotes the area of the image of the disk $\mathbb{D}(\gamma ; r(1-|\gamma|))$ under the mapping $g$. 


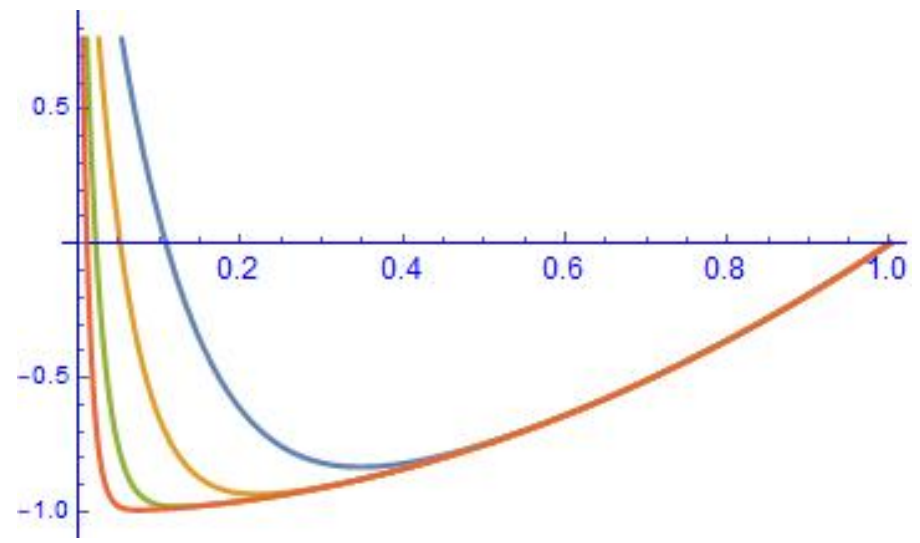

Figure 1. The roots $\gamma_{*}(m)$ of the equation $(1-\gamma)^{m}(3+\gamma)+\gamma^{2}-1=0$.

By applying Lemma 2.4, we obtain the following improved version of Theorem 1.2.

Theorem 2.5. For $0 \leq \gamma<1$ and $0 \leq \lambda \leq 512 / 243$, let $f \in \mathcal{B}\left(\Omega_{\gamma}\right)$ with $f(z)=\sum_{n=0}^{\infty} a_{n} z^{n}$ for $z \in \mathbb{D}$, then we have

$$
\sum_{n=0}^{\infty}\left|a_{n}\right| r^{n}+\left(\frac{8}{9}-\frac{27}{64} \lambda\right)\left(\frac{S_{r(1-\gamma)}}{\pi}\right)+\lambda\left(\frac{S_{r(1-\gamma)}}{\pi}\right)^{2} \leq 1 \quad \text { for } r \leq r_{0}=\frac{1+\gamma}{3+\gamma}
$$

Furthermore, the radius $r_{0}$ is sharp, and the bounds of $\lambda$ and 8/9 $-27 \lambda / 64$ cannot be improved.

Lemma 2.6. For $\gamma \in \mathbb{D}$, let $g \in \mathcal{B}(\mathbb{D})$ with $g(z)=\sum_{n=0}^{\infty} \alpha_{n}(z-\gamma)^{n}$, for $|z-\gamma| \leq$ $1-|\gamma|$, then

$$
|g(z)|+\sum_{n=N}^{\infty}\left|\alpha_{n}\right| \rho^{n} \leq 1, \quad \text { for } \rho \leq \rho_{N},
$$

where $\rho_{N}$ is the root of

$$
2(1+\gamma) \rho^{N}+(1+\gamma)(1-\gamma)^{N-1}(\rho-1)(1-\gamma-\rho)=0
$$

in $(0,1)$.

Using Lemma 2.6, we obtain the following Bohr-Rogosinski radius for the class $\mathcal{B}\left(\Omega_{\gamma}\right)$.

Theorem 2.7. For $0 \leq \gamma<1$ and integer $N(\geq 1)$, let $f \in \mathcal{B}\left(\Omega_{\gamma}\right)$ with $f(z)=$ $\sum_{n=0}^{\infty} a_{n} z^{n}$ for $z \in \mathbb{D}$. Then, we have

$$
\left|f\left(\frac{z-\gamma}{1-\gamma}\right)\right|+\sum_{n=N}^{\infty}\left|a_{n}\right| r^{n} \leq 1 \quad \text { for } r \leq r_{0}=\frac{\rho_{N}}{1-\gamma}
$$

where $\rho_{N}$ is the root of the equation

$$
2(1+\rho) \rho^{N}+(1+\gamma)(1-\gamma)^{N-1}(\rho-1)(1-\gamma-\rho)=0 .
$$

Furthermore, the constant $\rho_{N} /(1-\gamma)$ cannot be improved.

Using Lemma 1.5, we establish the following refined Bohr inequality for the class $\mathcal{B}\left(\Omega_{\gamma}\right)$. 
Theorem 2.9. For $0 \leq \gamma<1$, let $f \in \mathcal{B}\left(\Omega_{\gamma}\right)$ with $f(z)=\sum_{n=1}^{\infty} a_{n} z^{n}$ for $z \in \mathbb{D}$. Then we have

$$
\sum_{n=1}^{\infty}\left|a_{n}\right| r^{n}+\left(\frac{1}{1+\left|a_{1}\right|}+\frac{r}{1-r}\right) \sum_{n=2}^{\infty}\left|a_{n}\right|^{2} r^{2(n-1)} \leq 1 \quad \text { for } r \leq r_{0}=\frac{1+\gamma}{3+\gamma} .
$$

The constant $r_{0}$ cannot be improved.

\section{Proofs of the main results}

Proof of the Lemma 2.1. Without loss of generality, we may assume that $\gamma \in[0,1)$. Using Lemma 1.4, we obtain

$$
\sum_{n=1}^{\infty}\left|\alpha_{n}\right| \rho^{n} \leq \frac{1-\left|\alpha_{0}\right|^{2}}{1+\gamma} \sum_{n=1}^{\infty}\left(\frac{\rho}{1-\gamma}\right)^{n}=\frac{\left(1-\left|\alpha_{0}\right|^{2}\right) \rho}{(1+\gamma)(1-\gamma-\rho)} .
$$

Further, we have

$$
\sum_{n=1}^{\infty}\left|\alpha_{n}\right|^{m} \rho^{n} \leq \frac{\left(1-\left|\alpha_{0}\right|^{2}\right)^{m}}{(1+\gamma)^{m}} \sum_{n=1}^{\infty}\left(\frac{\rho}{(1-\gamma)^{m}}\right)^{n}=\frac{\left(1-\left|\alpha_{0}\right|^{2}\right)^{m} \rho}{(1+\gamma)^{m}\left((1-\gamma)^{m}-\rho\right)} .
$$

The series in (2.2) contains positive terms for $\beta \geq 0$. Our aim is to find the smallest value of $\gamma$ in $[0,1)$ for which $\beta \geq 0$. That is

$$
\beta=\frac{(1-\gamma)^{m}(3+\gamma)-\left(1-\gamma^{2}\right)}{8(m-1)}:=\frac{Q(\gamma)}{8(m-1)} \geq 0
$$

where $Q(\gamma)=(1-\gamma)^{m}(3+\gamma)-\left(1-\gamma^{2}\right)$. Clearly, $\gamma=1$ is a root of $Q(\gamma)$. Since $Q(\gamma)$ is a polynomial such that $Q(0)=2>0$ and $m \geq 2$, we have

$$
Q\left(\frac{9}{10}\right)=\frac{3.9}{10^{m}}+\frac{81}{100}-1 \leq \frac{84.9}{100}-1=-\frac{15.1}{100}<0 .
$$

Therefore, there exists at least one root of $Q(\gamma)$ in $(0,1)$. Let $\gamma_{*}$ be the smallest root of $Q(\gamma)$. Then, it is easy to see that $Q(\gamma) \geq 0$, and hence $\beta \geq 0$ for all $\gamma \in\left[0, \gamma_{*}\right]$. A simple computation using (3.1) and (3.2) shows that

$$
\begin{aligned}
& \left|\alpha_{0}\right|+\sum_{n=1}^{\infty}\left|\alpha_{n}\right| \rho^{n}+\beta \sum_{n=1}^{\infty}\left|\alpha_{n}\right|^{m} \rho^{n} \\
& \leq\left|\alpha_{0}\right|+\frac{\left(1-\left|\alpha_{0}\right|^{2}\right) \rho}{(1+\gamma)(1-\gamma-\rho)}+\beta \frac{\left(1-\left|\alpha_{0}\right|^{2}\right)^{m} \rho}{(1+\gamma)^{m}\left((1-\gamma)^{m}-\rho\right)} \\
& =1+\Psi_{\gamma}(\rho) \leq 1
\end{aligned}
$$

provided $\Psi_{\gamma}(\rho) \leq 0$, where

$$
\Psi_{\gamma}(\rho)=\frac{1-\left|\alpha_{0}\right|^{2}}{1+\gamma}\left(\frac{\rho}{1-\gamma-\rho}\right)+\beta\left(\frac{1-\left|\alpha_{0}\right|^{2}}{1+\gamma}\right)^{m}\left(\frac{\rho}{(1-\gamma)^{m}-\rho}\right)-\left(1-\left|\alpha_{0}\right|\right) .
$$

Since $(1-\gamma)-\rho>(1-\gamma)^{m}-\rho$, it is easy to see that $\Psi_{\gamma}(\rho)$ is an increasing function of $r$ for $r<(1-\gamma)^{m}$. A simplification shows that

$$
\begin{aligned}
& \Psi_{\gamma}(\rho) \\
& =K\left(1+\left(1-\left|\alpha_{0}\right|^{2}\right)^{m-1}\left(\frac{2 \beta \rho}{(1+\gamma)\left[(1-\gamma)^{m}-\rho\right]}+\frac{\phi_{\gamma}(\rho)}{\left(1-\left|\alpha_{0}\right|^{2}\right)^{m-1}}\right)-\frac{2}{1+\left|\alpha_{0}\right|}\right),
\end{aligned}
$$


where

$$
K=\frac{1-\left|\alpha_{0}\right|^{2}}{2} \text { and } \quad \phi_{\gamma}(\rho)=\frac{2 r}{(1+\gamma)(1-\gamma-\rho)}-1
$$

Let $\rho \leq \rho_{0}$ be such that $\Psi_{\gamma}(\rho) \leq \Psi_{\gamma}\left(\rho_{0}\right)$, and $\phi_{\gamma}\left(\rho_{0}\right)=0$. Then, it is easy to see that $\phi_{\gamma}\left(\rho_{0}\right)=0$ if, and only if, $\rho_{0}=\left(1-\gamma^{2}\right) /(3+\gamma)$. Therefore, it is enough to prove that $\Psi_{\gamma}\left(\rho_{0}\right) \leq 0$ for $\left|\alpha_{0}\right| \leq 1$. Let $\beta=\eta\left((1-\gamma)^{m}(3+\gamma)-\left(1-\gamma^{2}\right)\right)$, then it is easy to see

$$
\Psi_{\gamma}\left(\rho_{0}\right)=K\left(1+2 \eta\left(1-\left|\alpha_{0}\right|^{2}\right)^{m-1} \frac{1-\gamma^{2}}{(1+\gamma)^{m}}-\frac{2}{1+\left|\alpha_{0}\right|}\right):=K G_{\gamma}\left(\left|\alpha_{0}\right|\right),
$$

where

$$
G_{\gamma}(x)=1+2 \eta A(\gamma)\left(1-x^{2}\right)^{m-1}-\frac{2}{x+1}
$$

and

$$
A(\gamma)=\frac{1-\gamma^{2}}{(1+\gamma)^{m}}>0 \text { for } \gamma \in[0,1)
$$

It now remains to show that $G_{\gamma}(x) \leq 0$ for $\gamma \in[0,1)$ and $x \in[0,1]$. Since

$$
A^{\prime}(\gamma)=-\frac{2(1+\gamma) \gamma+m\left(1-\gamma^{2}\right)}{(1+\gamma)^{m+1}} \leq 0, \quad \text { for } \gamma \in[0,1)
$$

and $A(0)=1, \quad A(1)=0$, it follows that $A(\gamma)$ is a decreasing function and hence $A(\gamma) \leq A(0)=1$. Since $x \leq 1$ and $0<A(\gamma) \leq 1$, we have

$$
-A(\gamma) x(1+x)^{2}\left(1-x^{2}\right)^{m-2}>-4
$$

From (3.4), we have

$$
\begin{aligned}
\left(G_{\gamma}(x)\right)^{\prime} & =\frac{2}{(1+x)^{2}}\left(1-2 \eta A(\gamma)(m-1) x(1+x)^{2}\left(1-x^{2}\right)^{m-2}\right) \\
& \geq \frac{2(1-8(m-1) \eta)}{(1+x)^{2}} .
\end{aligned}
$$

Clearly, $\left(G_{\gamma}(x)\right)^{\prime}>0$ for $x \in(0,1)$ whenever $\eta \leq 1 /(8(m-1))$. Therefore, $G_{\gamma}(x)$ is an increasing function on $[0,1]$ for $\eta \leq 1 /(8(m-1))$. Equivalently,

$$
\beta \leq \frac{(1-\gamma)^{m}(3+\gamma)-\left(1-\gamma^{2}\right)}{8(m-1)}
$$

In particular, $G_{\gamma}(x) \leq 0$ for $\gamma \in\left[0, \gamma_{*}\right]$ and $x \in[0,1]$, where $\gamma_{*}$ is the smallest root of the equation $(1-\gamma)^{m}(3+\gamma)-\left(1-\gamma^{2}\right)=0$. This completes the proof.

Proof of Theorem 2.3. For $0 \leq \gamma<1$, let

$$
\Omega_{\gamma}=\left\{z \in \mathbb{C}:\left|z+\frac{\gamma}{1-\gamma}\right|<\frac{1}{1-\gamma}\right\}
$$

and the function $f: \Omega_{\gamma} \rightarrow \mathbb{D}$ be given by $f(z)=\sum_{n=0}^{\infty} a_{n} z_{n}$. Then the function $g$ defined by

$$
g(z)=f\left(\frac{z-\gamma}{1-\gamma}\right)=\sum_{n=0}^{\infty} \frac{a_{n}}{(1-\gamma)^{n}}(z-\gamma)^{n} \quad \text { for }|z-\gamma|<1-\gamma
$$

belongs to $\mathcal{B}(\mathbb{D})$. Applying Lemma 2.1 to the function $g$, we obtain

$$
\left|a_{0}\right|+\sum_{n=1}^{\infty}\left(\frac{\left|a_{n}\right|}{(1-\gamma)^{n}}+\beta\left(\frac{\left|a_{n}\right|}{(1-\gamma)^{n}}\right)^{m}\right) \rho^{n} \leq 1 \quad \text { for } \rho \leq \rho_{0}=\frac{1-\gamma^{2}}{3+\gamma}
$$


That is

$$
\left|a_{0}\right|+\sum_{n=1}^{\infty}\left(\left|a_{n}\right|+\beta \frac{\left|a_{n}\right|^{m}}{(1-\gamma)^{(m-1) n}}\right)\left(\frac{\rho}{1-\gamma}\right)^{n} \leq 1 \quad \text { for } \rho \leq \rho_{0}=\frac{1-\gamma^{2}}{3+\gamma}
$$

which is equivalent to

$$
\left|a_{0}\right|+\sum_{n=1}^{\infty}\left(\left|a_{n}\right|+\beta \frac{\left|a_{n}\right|^{m}}{(1-\gamma)^{(m-1) n}}\right) r^{n} \leq 1 \quad \text { for } r \leq r_{0}=\frac{1+\gamma}{3+\gamma},
$$

where $\rho=r(1-\gamma)$ and

$$
\beta=\frac{(1-\gamma)^{m}(3+\gamma)-\left(1-\gamma^{2}\right)}{8(m-1)} \text { for } 0 \leq \gamma \leq \gamma_{*}<1
$$

Here $\gamma_{*}$ is the smallest root of the equation $(1-\gamma)^{m}(3+\gamma)+\gamma^{2}-1=0$.

In order to prove the sharpness of the radius, we consider the composition function $f_{a}=h \circ H$ which maps $\Omega_{\gamma}$ univalently onto $\mathbb{D}$, where $H: \Omega_{\gamma} \rightarrow \mathbb{D}$ defined by $H(z)=(1-\gamma) z+\gamma$ and $h: \mathbb{D} \rightarrow \mathbb{D}$ with $h(z)=(a-z) /(1-a z)$, for $a \in(0,1)$. A simple computation shows that

$$
f_{a}(z)=\frac{a-\gamma-(1-\gamma) z}{1-a \gamma-a(1-\gamma) z}=C_{0}-\sum_{n=1}^{\infty} C_{n} z^{n} \quad \text { for } \quad z \in \mathbb{D}
$$

where $a \in(0,1)$ and

$$
C_{0}=\frac{a-\gamma}{1-a \gamma} \quad \text { and } \quad C_{n}=\frac{1-a^{2}}{a(1-a \gamma)}\left(\frac{a(1-\gamma)}{1-a \gamma}\right)^{n} .
$$

A simple computation shows that

$$
\begin{aligned}
& \left|C_{0}\right|+\sum_{n=1}^{\infty}\left(\left|C_{n}\right|+\beta \frac{\left|C_{n}\right|^{m}}{(1-\gamma)^{(m-1) n}}\right) r^{n}=\frac{a-\gamma}{1-a \gamma}+\sum_{n=1}^{\infty}\left(\frac{1-a^{2}}{a(1-a \gamma)}\left(\frac{a(1-\gamma)}{1-a \gamma}\right)^{n}\right. \\
& \left.\quad+\frac{\beta}{(1-\gamma)^{(m-1) n}} \frac{\left(1-a^{2}\right)^{m}}{a^{m}(1-a \gamma)^{m}}\left(\frac{a(1-\gamma)}{1-a \gamma}\right)^{m n}\right) r^{n} \\
& =\frac{a-\gamma}{1-a \gamma}+\frac{(1+a)(1-a)(1-\gamma) r}{(1-a \gamma)((1-a \gamma)-a r(1-\gamma))}+\frac{\beta(1-a)^{m}(1+a)^{m}(1-\gamma) r}{(1-a \gamma)^{m}\left((1-a \gamma)^{m}-a^{m}(1-\gamma) r\right)} \\
& =1-(1-a) \Phi_{\gamma}(r),
\end{aligned}
$$

where

$$
\begin{aligned}
\Phi_{\gamma}(r)= & -\frac{(1+a)(1-\gamma) r}{(1-a \gamma)((1-a \gamma)-a r(1-\gamma))}-\frac{\beta(1-a)^{m-1}(1+a)^{m}(1-\gamma) r}{(1-a \gamma)^{m}\left((1-a \gamma)^{m}-a^{m}(1-\gamma) r\right)} \\
& -\frac{1}{1-a}\left(\frac{a-\gamma}{1-a \gamma}-1\right) \\
= & -\frac{(1+a)(1-\gamma) r}{(1-a \gamma)((1-a \gamma)-a r(1-\gamma))}-\frac{\beta(1-a)^{m-1}(1+a)^{m}(1-\gamma) r}{(1-a \gamma)^{m}\left((1-a \gamma)^{m}-a^{m}(1-\gamma) r\right)} \\
& +\frac{1+\gamma}{1-a \gamma}
\end{aligned}
$$

We note that for $r \in(0,1)$,

$$
\Phi_{\gamma}^{\prime}(r)=-\frac{(1+a)(1-\gamma)}{((1-a \gamma)-a r(1-\gamma))^{2}}-\frac{\beta(1-a)^{m-1}(1+a)^{m}(1-\gamma)}{\left((1-a \gamma)^{m}-a^{m}(1-\gamma) r\right)^{2}}<0 .
$$


Therefore, $\Phi_{\gamma}(r)$ is strictly decreasing function of $r$ in $(0,1)$. Hence, for $r>r_{0}=$ $(1+\gamma) /(3+\gamma)$, we have $\Phi_{\gamma}(r)<\Phi_{\gamma}\left(r_{0}\right)$. A simple computation shows that

$$
\lim _{a \rightarrow 1} \Phi_{\gamma}\left(r_{0}\right)=-\frac{2 r_{0}}{(1-\gamma)\left(1-r_{0}\right)}+\frac{1+\gamma}{1-\gamma}=0 .
$$

Thus, $\Phi_{\gamma}(r)<0$ for $r>r_{0}$. Hence, $1-(1-a) \Phi_{\gamma}(r)>1$ for $r>r_{0}$, which shows that $r_{0}$ is the best possible. This completes the proof.

Proof of Lemma 2.4. Without loss of generality, we assume that $\gamma \in[0,1)$. Also let $z \in \mathbb{D}_{\gamma}:=\mathbb{D}(\gamma ; 1-\gamma)$ if, and only if, $\phi=(z-\gamma) /(1-\gamma) \in \mathbb{D}$. Then we have

$$
g(z)=\sum_{n=0}^{\infty} \alpha_{n}(1-\gamma)^{n} \phi^{n}(z)=\sum_{n=0}^{\infty} b_{n} \phi^{n}(z):=G(\phi(z))
$$

for $z \in \mathbb{D}_{\gamma}$, where $b_{n}=\alpha_{n}(1-\gamma)^{n}$. It is known that for arbitrary function $H(z)=$ $\sum_{n=0}^{\infty} h_{n} z^{n}, z \in \mathbb{D}$, the area functional

$$
\frac{S_{r}}{\pi}=\operatorname{Area}(H(\mathbb{D}(0, r)))=\frac{1}{\pi} \iint_{|z|<r}\left|H^{\prime}(z)\right|^{2} d x d y \leq \sum_{n=1}^{\infty} n\left|h_{n}\right|^{2} r^{2 n} .
$$

A simple computation shows that

$$
\frac{S_{\rho}^{\gamma}}{\pi}=\frac{1}{\pi} \operatorname{Area}(G(\mathbb{D}(0, \rho))) \leq\left(1-\left|b_{0}\right|^{2}\right)^{2} \frac{\rho^{2}}{\left(1-\rho^{2}\right)^{2}}=\left(1-\left|\alpha_{0}\right|^{2}\right)^{2} \frac{\rho^{2}}{\left(1-\rho^{2}\right)^{2}}
$$

Using Lemma 1.4, we deduce that

$$
\sum_{n=1}^{\infty}\left|\alpha_{n}\right| \rho^{n} \leq \frac{1-\left|\alpha_{0}\right|^{2}}{1+\gamma} \sum_{n=1}^{\infty}\left(\frac{\rho}{1-\gamma}\right)^{n}=\frac{1-\left|\alpha_{0}\right|^{2}}{1+\gamma} \frac{\rho}{1-\gamma-\rho} .
$$

In view of (3.5) and (3.6), we obtain

$$
\begin{aligned}
& \left|\alpha_{0}\right|+\sum_{n=1}^{\infty}\left|\alpha_{n}\right| \rho^{n}+k\left(\frac{S_{\rho}^{\gamma}}{\pi}\right)+\lambda\left(\frac{S_{\rho}^{\gamma}}{\pi}\right)^{2} \\
& =\left|\alpha_{0}\right|+\frac{\left(1-\left|\alpha_{0}\right|^{2}\right) \rho}{(1+\gamma)(1-\gamma-\rho)}+k \frac{\left(1-\left|\alpha_{0}\right|^{2}\right)^{2} \rho^{2}}{\left(1-\rho^{2}\right)^{2}}+\lambda \frac{\left(1-\left|\alpha_{0}\right|^{2}\right)^{4} \rho^{4}}{\left(1-\rho^{2}\right)^{4}} \\
& =1+\Psi_{1}^{\gamma}(\rho)
\end{aligned}
$$

where

$$
\Psi_{1}^{\gamma}(\rho)=\frac{\left(1-\left|\alpha_{0}\right|^{2}\right) \rho}{(1+\gamma)(1-\gamma-\rho)}+k \frac{\left(1-\left|\alpha_{0}\right|^{2}\right)^{2} \rho^{2}}{\left(1-\rho^{2}\right)^{2}}+\lambda \frac{\left(1-\left|\alpha_{0}\right|^{2}\right)^{4} \rho^{4}}{\left(1-\rho^{2}\right)^{4}}-\left(1-\left|\alpha_{0}\right|\right)
$$

which can be written as

$$
\begin{aligned}
\Psi_{1}^{\gamma}(\rho)= & \frac{1-\left|\alpha_{0}\right|^{2}}{2}\left(1+2 \lambda\left(1-\left|\alpha_{0}\right|^{2}\right)^{3}\left(\left(\frac{\rho^{4}}{\left(1-\rho^{2}\right)^{4}}+\frac{k}{\lambda} \frac{\rho^{2}}{\left(1-\rho^{2}\right)^{2}\left(1-\left|\alpha_{0}\right|^{2}\right)^{2}}\right)\right.\right. \\
& \left.\left.+\frac{1}{2 \lambda\left(1-\left|\alpha_{0}\right|^{2}\right)^{3}}\left(\frac{2 \rho}{(1+\gamma)(1-\gamma-\rho)}-1\right)\right)-\frac{2}{1+\left|\alpha_{0}\right|}\right) .
\end{aligned}
$$

We note that

$$
\begin{aligned}
\left(\Psi_{1}^{\gamma}\right)^{\prime}(\rho)= & \frac{\left(1-\left|\alpha_{0}\right|^{2}\right)}{(1+\gamma)} \frac{1-\gamma}{((1-\gamma-\rho))^{2}}+k \frac{\left(1-\left|\alpha_{0}\right|^{2}\right)^{2}\left(2 \rho\left(1-\rho^{2}\right)^{2}+4 \rho^{3}\left(1-\rho^{2}\right)\right)}{\left(1-\rho^{2}\right)^{4}} \\
& +\lambda \frac{\left(1-\left|\alpha_{0}\right|^{2}\right)^{4} 4 \rho^{3}\left(1-\rho^{2}\right)^{4}+8 \rho^{5}\left(1-\rho^{2}\right)^{3}}{\left(1-\rho^{2}\right)^{8}}>0 .
\end{aligned}
$$



where

Therefore, $\Psi_{1}^{\gamma}(\rho)$ is an increasing function and hence $\Psi_{1}^{\gamma}(\rho) \leq \Psi_{1}^{\gamma}\left(\rho_{0}\right)$ for $\rho \leq \rho_{0}$,

$$
\frac{2 \rho_{0}}{(1+\gamma)\left(1-\gamma-\rho_{0}\right)}=1, \quad \text { i.e., } \quad \rho_{0}=\frac{1-\gamma^{2}}{3+\gamma}
$$

A simple computation shows that

$$
\begin{aligned}
\Psi_{1}^{\gamma}\left(\rho_{0}\right) & =\frac{1-\left|\alpha_{0}\right|^{2}}{2}\left(1+2 \lambda\left(1-\left|\alpha_{0}\right|^{2}\right)^{3} A^{4}(\gamma)+2 k\left(1-\left|\alpha_{0}\right|^{2}\right) A^{2}(\gamma)-\frac{2}{1+\left|\alpha_{0}\right|}\right) \\
& =\frac{1-\left|\alpha_{0}\right|^{2}}{2} J\left(\left|\alpha_{0}\right|\right),
\end{aligned}
$$

where

$$
J(x)=1+2 \lambda\left(1-x^{2}\right)^{3} A^{4}(\gamma)+2 k\left(1-x^{2}\right) A^{2}(\gamma)-\frac{2}{1+x} \quad \text { for } \quad x \in[0,1]
$$

and

$$
A(\gamma)=\frac{(3+\gamma)\left(1-\gamma^{2}\right)}{(3+\gamma)^{2}-\left(1-\gamma^{2}\right)^{2}}
$$

It is enough to show that $J(x) \leq 0$ for $x \in[0,1]$ and $\gamma \in[0,1)$ so that $\Psi_{1}^{\gamma}\left(\rho_{0}\right) \leq 0$. We note that $A(\gamma)>0$ for $\gamma \in[0,1)$. Further,

$$
J(0)=2 \lambda A^{4}(\gamma)+2 k A^{2}(\gamma)-1, \quad \text { and } \quad \lim _{x \rightarrow 1^{-}} J(x)=0 .
$$

It can be seen that $A(\gamma)=\left(f_{1} \circ f_{2}\right)(\gamma)$, where $f_{1}(\rho)=\rho /\left(1-\rho^{2}\right)$ and $f_{2}(\gamma)=$ $\left(1-\gamma^{2}\right) /(3+\gamma)$. Since $A^{\prime}(\gamma)=f_{1}^{\prime}\left(f_{2}(\gamma)\right) f_{2}^{\prime}(\gamma)$, where

$$
f_{2}^{\prime}(\gamma)=-\left(\frac{\gamma^{2}+6 \gamma+1}{(3+\gamma)^{2}}\right)<0
$$

which implies that $f_{1}(\rho)$ is an increasing function of $\rho$ in $(0,1)$, and $f_{2}$ is a decreasing function of $\gamma$ in $[0,1)$. Hence, it follows that $A(\gamma)$ is a decreasing function of $\gamma$ in $[0,1)$, with $A(0)=3 / 8$ and $A(1)=0$. It can be seen that $A^{2}(\gamma)$ and $A^{4}(\gamma)$ are decreasing functions on $[0,1)$. Therefore, we have

$$
A^{2}(\gamma) \leq A^{2}(0)=\frac{9}{64} \quad \text { and } \quad A^{4}(\gamma) \leq A^{4}(0)=\frac{81}{4096} .
$$

Since $x \in[0,1]$, we have

$$
x(1+x)^{2} A^{2}(\gamma) \leq \frac{9}{16} \quad \text { and } \quad x(1+x)^{2}\left(1-x^{2}\right)^{2} A^{4}(\gamma) \leq \frac{81}{1024} .
$$

As a consequence, we obtain

$$
\begin{aligned}
J^{\prime}(x) & =\frac{2}{(1+x)^{2}}\left(1-2 k x(1+x)^{2} A^{2}(\gamma)-6 \lambda x(1+x)^{2}\left(1-x^{2}\right)^{2} A^{4}(\gamma)\right) \\
& \geq \frac{2}{(1+x)^{2}}\left(1-\left(\frac{9 k}{8}+\frac{243 \lambda}{512}\right)\right) \\
& \geq 0, \quad \text { if } 9 k / 8+243 \lambda / 512 \leq 1 .
\end{aligned}
$$

Since $0 \leq \lambda \leq 512 / 243$ and $k \geq 0$, therefore, $J(x)$ is an increasing function in $[0,1]$ for $k+27 \lambda / 64 \leq 8 / 9$. Hence, $J(x) \leq 0$ for all $x \in[0,1]$ and $\gamma \in[0,1)$. This completes the proof. 
Proof of Theorem 2.5. Let $f \in \mathcal{B}\left(\Omega_{\gamma}\right)$ and $g(z)=f((z-\gamma) /(1-\gamma))$. Then, it is easy to see that $g \in \mathcal{B}(\mathbb{D})$ and

$$
g(z)=\sum_{n=0}^{\infty} \frac{a_{n}}{(1-\gamma)^{n}}(z-\gamma)^{n} .
$$

Using Lemma 2.4, we obtain

$$
\sum_{n=0}^{\infty} \frac{\left|a_{n}\right|}{(1-\gamma)^{n}} \rho^{n}+\left(\frac{8}{9}-\frac{27}{64} \lambda\right)\left(\frac{S_{\rho}^{\gamma}}{\pi}\right)+\lambda\left(\frac{S_{\rho}^{\gamma}}{\pi}\right)^{2} \leq 1 \quad \text { for } \rho \leq \frac{1-\gamma^{2}}{3+\gamma}
$$

which is equivalent to

$$
\sum_{n=0}^{\infty}\left|a_{n}\right|\left(\frac{\rho}{(1-\gamma)}\right)^{n}+\left(\frac{8}{9}-\frac{27}{64} \lambda\right)\left(\frac{S_{\rho}^{\gamma}}{\pi}\right)+\lambda\left(\frac{S_{\rho}^{\gamma}}{\pi}\right)^{2} \leq 1 \quad \text { for } \rho \leq \frac{1-\gamma^{2}}{3+\gamma}
$$

Set $\rho=r(1-\gamma)$, then in view of (3.8), we obtain

$$
\sum_{n=0}^{\infty}\left|a_{n}\right| r^{n}+\left(\frac{8}{9}-\frac{27}{64} \lambda\right)\left(\frac{S_{r(1-\gamma)}^{\gamma}}{\pi}\right)+\lambda\left(\frac{S_{r(1-\gamma)}^{\gamma}}{\pi}\right)^{2} \leq 1 \quad \text { for } r \leq \frac{1+\gamma}{3+\gamma} .
$$

To show the sharpness of the result, we consider the following function

$$
f_{a}(z)=\frac{a-\gamma-(1-\gamma) z}{1-a \gamma-a(1-\gamma) z} \text { for } z \in \Omega_{\gamma} \text { and } a \in(0,1) .
$$

Define $\phi: \mathbb{D} \rightarrow \mathbb{D}$ by $\phi(z)=(a-z) /(1-a z)$ and $H: \Omega_{\gamma} \rightarrow \mathbb{D}$ by $H(z)=(1-\gamma) z+\gamma$. Then, the function $f_{a}=\phi \circ H$ maps $\Omega_{\gamma}$, univalently onto $\mathbb{D}$. A simple computation shows that

$$
f_{a}(z)=\frac{a-\gamma-(1-\gamma) z}{1-a \gamma-a(1-\gamma) z}=C_{0}-\sum_{n=1}^{\infty} C_{n} z^{n} \quad \text { for } \quad z \in \mathbb{D}
$$

where $a \in(0,1)$ and

$$
C_{0}=\frac{a-\gamma}{1-a \gamma} \quad \text { and } \quad C_{n}=\frac{1-a^{2}}{a(1-a \gamma)}\left(\frac{a(1-\gamma)}{1-a \gamma}\right)^{n} .
$$

A simple computation using (3.9) shows that

$$
\begin{aligned}
\sum_{n=0}^{\infty}\left|C_{n}\right| r^{n}+\left(\frac{8}{9}-\frac{27}{64} \lambda\right)\left(\frac{S_{r(1-\gamma)}^{\gamma}}{\pi}\right)+\lambda\left(\frac{S_{r(1-\gamma)}^{\gamma}}{\pi}\right)^{2} \\
=\frac{a-\gamma}{1-a \gamma}+\left(\frac{1-a^{2}}{1-a \gamma}\right) \frac{(1-\gamma) r}{1-a \gamma-a r(1-\gamma)} \\
\quad+\left(\frac{8}{9}-\frac{27}{64} \lambda\right) \frac{r^{2}\left(1-a^{2}\right)^{2}(1-\gamma)^{4}}{\left((1-a \gamma)^{2}-a^{2} r^{2}(1-\gamma)^{4}\right)^{2}}+\lambda \frac{r^{4}\left(1-a^{2}\right)^{4}(1-\gamma)^{8}}{\left((1-a \gamma)^{2}-a^{2} r^{2}(1-\gamma)^{4}\right)^{4}} \\
:=1-(1-a) \Phi_{1}^{\gamma}(r),
\end{aligned}
$$

where

$$
\begin{aligned}
\Phi_{1}^{\gamma}(r)= & -\frac{(1+a)(1-\gamma) r}{(1-a \gamma-a r(1-\gamma))(1-a \gamma)}-\left(\frac{8}{9}-\frac{27}{64} \lambda\right) \frac{r^{2}(1-a)(1+a)^{2}(1-\gamma)^{4}}{\left((1-a \gamma)^{2}-a^{2} r^{2}(1-\gamma)^{4}\right)^{2}} \\
& -\lambda \frac{r^{4}(1-a)^{3}(1+a)^{4}(1-\gamma)^{8}}{\left((1-a \gamma)^{2}-a^{2} r^{2}(1-\gamma)^{4}\right)^{4}}-\frac{1}{1-a}\left(\frac{a-\gamma}{1-a \gamma}-1\right) .
\end{aligned}
$$


A simple computation shows that

$$
\begin{aligned}
\left(\Phi_{1}^{\gamma}\right)^{\prime}(r)= & -\frac{(1+a)(1-\gamma)}{(1-a \gamma-a r(1-\gamma))^{2}}-\left(\frac{8}{9}-\frac{27}{64} \lambda\right) \frac{2 r\left((1-a \gamma)^{2}+a^{2} r^{2}(1-\gamma)^{4}\right)}{\left((1-a \gamma)^{2}-a^{2} r^{2}(1-\gamma)^{4}\right)^{3}} \\
& -\lambda \frac{4 r^{3}(1-a)^{3}(1+a)^{4}(1-\gamma)^{8}\left((1-a \gamma)^{2}+a^{2} r^{2}(1-\gamma)^{4}\right)}{\left((1-a \gamma)^{2}-a^{2} r^{2}(1-\gamma)^{4}\right)^{5}}<0
\end{aligned}
$$

for $r$ in $(0,1)$ and hence $\Phi_{1}^{\gamma}(r)$ is strictly decreasing function of $r$. Therefore, for $r>r_{0}=(1+\gamma) /(3+\gamma)$, we have $\Phi_{1}^{\gamma}(r)<\Phi_{1}^{\gamma}\left(r_{0}\right)$. An elementary calculation shows that

$$
\lim _{a \rightarrow 1} \Phi_{1}^{\gamma}\left(r_{0}\right)=-\frac{2 r_{0}}{(1-\gamma)\left(1-r_{0}\right)}+\frac{1+\gamma}{1-\gamma}=0 .
$$

Therefore, $\Phi_{1}^{\gamma}(r)<0$ for $r>r_{0}$. Hence, $1-(1-a) \Phi_{\gamma}(r)>1$ for $r>r_{0}$, which shows that $r_{0}$ is the best possible.

Proof of Lemma 2.6. Let $g \in \mathcal{B}(\mathbb{D})$. Then by the Schwarz-Pick lemma, for any $g \in \mathcal{B}(\mathbb{D})$, we have

$$
|g(z)| \leq \frac{\rho+|g(\gamma)|}{1+\rho|g(\gamma)|}=\frac{\rho+\left|\alpha_{0}\right|}{1+\rho\left|\alpha_{0}\right|} \quad \text { for } \quad z \in \mathbb{D} .
$$

For functions $g \in \mathcal{B}(\mathbb{D})$, from Lemma 1.4, we have

$$
\left|\alpha_{n}\right| \leq(1+|\gamma|)^{n-1} \frac{1-\left|\alpha_{0}\right|^{2}}{\left(1-|\gamma|^{2}\right)^{n}} \quad \text { for } n \geq 1
$$

A simple computation using (3.11) gives

$$
\sum_{n=N}^{\infty}\left|\alpha_{n}\right| \rho^{n} \leq \frac{1-\left|\alpha_{0}\right|^{2}}{1+\gamma} \sum_{n=N}^{\infty}\left(\frac{\rho}{1-\gamma}\right)^{n}=\frac{\left(1-\left|\alpha_{0}\right|^{2}\right)}{(1+\gamma)(1-\gamma)^{N-1}}\left(\frac{\rho^{N}}{1-\gamma-\rho}\right) .
$$

From (3.10) and (3.12) we obtain

$$
\begin{aligned}
|g(z)|+\sum_{n=N}^{\infty}\left|\alpha_{n}\right| \rho^{n} & \leq \frac{\rho+\left|\alpha_{0}\right|}{1+\rho\left|\alpha_{0}\right|}+\frac{\left(1-\left|\alpha_{0}\right|^{2}\right)}{(1+\gamma)(1-\gamma)^{N-1}}\left(\frac{\rho^{N}}{1-\gamma-\rho}\right) \\
& =1+\frac{\Phi_{N}^{\gamma}(\rho)}{\left(1+\rho\left|\alpha_{0}\right|\right)(1+\gamma)(1-\gamma)^{N-1}(1-\gamma-\rho)}
\end{aligned}
$$

where

$$
\begin{aligned}
\Phi_{N}^{\gamma}(\rho)= & \left(\rho+\left|\alpha_{0}\right|\right) A(\gamma)(1-\gamma-\rho)+\left(1+\left|\alpha_{0}\right|\right)\left(1-\left|\alpha_{0}\right|\right)\left(1+\rho\left|\alpha_{0}\right|\right) \rho^{N} \\
& -\left(1+\rho\left|\alpha_{0}\right|\right) A(\gamma)(1-\gamma-\rho) \\
= & \left(1-\left|\alpha_{0}\right|\right)\left(\left(1+\left|\alpha_{0}\right|\right)\left(1+\rho\left|\alpha_{0}\right|\right) \rho^{N}+A(\gamma)(\rho-1)(1-\gamma-\rho)\right) \\
\leq & \left(1-\left|\alpha_{0}\right|\right)\left(2(1+\gamma) \rho^{N}+A(\gamma)(\rho-1)(1-\gamma-\rho)\right)
\end{aligned}
$$

where $A(\gamma)=(1+\gamma)(1-\gamma)^{N-1}$ and $\left|\alpha_{0}\right| \leq 1$. An observation shows that $\Phi_{N}^{\gamma}(\rho) \leq 0$ if $2(1+\gamma) \rho^{N}+A(\gamma)(\rho-1)(1-\gamma-\rho) \leq 0$, and this holds for $\rho \leq \rho_{N}$, where $\rho_{N}$ is the root of

$$
F_{N}(\gamma, \rho)=2(1+\gamma) \rho^{N}+A(\gamma)(\rho-1)(1-\gamma-\rho)=0
$$

The existence of the root $\rho_{N}$ in $(0,1)$ follows from the fact that $F_{N}(\gamma, \rho)$ is continuous and $F_{N}(\gamma, 0) F_{N}(\gamma, 1)<0$. 
Proof of Theorem 2.7. For $0 \leq \gamma<1$, let $f \in \mathcal{B}\left(\Omega_{\gamma}\right)$ such that $f(z)=\sum_{n=0}^{\infty} a_{n} z^{n}$ for $z \in \mathbb{D}$. Then, it is easy to see that

$$
g(z)=f\left(\frac{z-\gamma}{1-\gamma}\right) \in \mathcal{B}(\mathbb{D}) \quad \text { for }|z-\gamma|<1-|\gamma|
$$

Further,

$$
g(z)=f\left(\frac{z-\gamma}{1-\gamma}\right)=\sum_{n=0}^{\infty} \frac{a_{n}}{(1-\gamma)^{n}}(z-\gamma)^{n} .
$$

An application of Lemma 2.6 shows that

$$
\left|f\left(\frac{z-\gamma}{1-\gamma}\right)\right|+\sum_{n=N}^{\infty} \frac{\left|a_{n}\right|}{(1-\gamma)^{n}} \rho^{n} \leq 1 \quad \text { for } \rho \leq \rho_{N} .
$$

Since $|z-\gamma|<1-\gamma$, we set $z-\gamma=w(1-\gamma)$ for some $w \in \mathbb{D}$ and $\rho=r(1-\gamma)$. Then, from (3.14), we obtain

$$
|f(w)|+\sum_{n=N}^{\infty}\left|a_{n}\right| r^{n} \leq 1 \quad \text { for } r \leq \frac{\rho_{N}}{1-\gamma},
$$

where $\rho_{N}$ as in Lemma 2.6. That is, $\rho_{N}$ is the smallest root of the equation $2(1+$ $\rho) \rho^{N}+A(\gamma)(\rho-1)(1-\gamma-\rho)=0$.

In order to show the sharpness of the result, we consider the following function $f_{a}$ defined by

$$
f_{a}(z)=\frac{1-\gamma-(1-\gamma) z}{(1-a \gamma)-(1-\gamma) z}=C_{0}-\sum_{n=1}^{\infty} C_{n} z^{n} \quad \text { for } \quad z \in \mathbb{D}
$$

For $\gamma \in[0,1), a>\gamma$ and $\rho=r(1-\gamma)$, we obtain

$$
\begin{aligned}
& M:=\left|f_{a}(-\rho)\right|+\sum_{n=N}^{\infty}\left|C_{n}\right| \rho^{n} \\
& =\frac{(a-\gamma)+(1-\gamma) \rho}{(1-a \gamma)+a(1-\gamma) \rho}+\frac{1-a^{2}}{a(1-a \gamma)}\left(\frac{a(1-\gamma)}{1-a \gamma}\right)^{N} \rho^{N}\left(\frac{1-a \gamma}{(1-a \gamma)-a(1-\gamma) \rho}\right) \\
& =\frac{(a-\gamma)+(1-\gamma) \rho}{(1-a \gamma)+a(1-\gamma) \rho}+\frac{\left(1-a^{2}\right) B^{N} \rho^{N}}{a((1-a \gamma)-a(1-\gamma) \rho)}, \quad \text { where } B=\frac{a(1-\gamma)}{1-a \gamma} \\
& =\frac{((a-\gamma)+(1-\gamma) \rho)((1-a \gamma)-a(1-\gamma) \rho)+d \rho^{N}\left(1-a^{2}\right)((1-a \gamma)+a(1-\gamma) \rho)}{((1-a \gamma)+a(1-\gamma) \rho)((1-a \gamma)-a(1-\gamma) \rho)} \\
& =1+\frac{V(\rho)}{((1-a \gamma)+a(1-\gamma) \rho)((1-a \gamma)-a(1-\gamma) \rho)},
\end{aligned}
$$

where $d=B^{N} / a$ and

$$
\begin{aligned}
V(\rho):= & ((a-\gamma)+(1-\gamma) \rho)((1-a \gamma)-a(1-\gamma) \rho) \\
& +d \rho^{N}\left(1-a^{2}\right)((1-a \gamma)+a(1-\gamma) \rho) \\
& -((1-a \gamma)+a(1-\gamma) \rho)((1-a \gamma)-a(1-\gamma) \rho) \\
= & (1-a)\left((1+a)((1-a \gamma)+a(1-\gamma) \rho) d \rho^{N}\right. \\
& +((1-a \gamma)-a(1-\gamma) \rho)(\rho(1-\gamma)-(1+\gamma))) .
\end{aligned}
$$


From (3.15), it is easy to see that $M>1$ if $V(\rho)>0$. Note that $V(\rho)>0$ if

$$
\begin{aligned}
W_{a}(\rho):= & (1+a)((1-a \gamma)+a(1-\gamma) \rho) d \rho^{N} \\
& +((1-a \gamma)-a(1-\gamma) \rho)(\rho(1-\gamma)-(1+\gamma)) .
\end{aligned}
$$

Allowing $a \rightarrow 1$, from the inequality (3.16), it can be seen that

$$
W_{1}(\rho)=2(1-\gamma)(1+\rho) \rho^{N}+(1-\gamma)(1-\rho)(\rho(1-\gamma)-(1+\gamma)) .
$$

Now for $\rho>\rho_{N}$ and $0<\gamma<1$, we obtian

$$
W_{1}(\rho)>(1-\gamma)\left(2\left(1+\rho_{N}\right) \rho_{N}^{N}+(1+\gamma)(1-\gamma)^{N-1}\left(\rho_{N}-1\right)\left(1-\gamma-\rho_{N}\right)\right)
$$

Since $\rho_{N}$ is a root of (3.13), we have

$$
2(1+\gamma) \rho_{N}^{N}+A(\gamma)\left(\rho_{N}-1\right)\left(1-\gamma-\rho_{N}\right)=0 .
$$

In view of (3.17) and (3.18), it is easy to see that $W_{1}(\rho)>0$ for $\rho>\rho_{N}$. Thus, $M>1$ if $r>\rho_{N} /(1-\gamma)$. This proves the sharpness.

Proof of Theorem 2.9. Let $f \in \mathcal{B}\left(\Omega_{\gamma}\right)$ be given by $f(z)=\sum_{n=1}^{\infty} a_{n} z^{n}$ for $z \in \mathbb{D}$. Then, $f$ can be expressed as $f(z)=z h(z)$, where $h \in B\left(\Omega_{\gamma}\right)$ with $h(z)=\sum_{n=0}^{\infty} b_{n} z^{n}$ and $b_{n}=a_{n+1}$. Let $\left|b_{0}\right|=\left|a_{1}\right|=a$, and $h_{0}(z)=g(z)-b_{0}$. Using Lemma 1.5, we obtain

$$
\begin{aligned}
& \sum_{n=0}^{\infty}\left|b_{n}\right| r^{n}+\left(\frac{1}{1+\left|b_{0}\right|}+\frac{r}{1+r}\right) \sum_{n=1}^{\infty}\left|b_{n}\right|^{2} r^{2 n} \\
& \leq a+\frac{1-a^{2}}{1+\gamma} \frac{r}{1-r}+\left(\frac{1}{1+a}+\frac{r}{1-r}\right)\left(\frac{1-a^{2}}{1+\gamma}\right)^{2} \frac{r^{2}}{1-r^{2}}
\end{aligned}
$$

That is,

$$
\begin{aligned}
\sum_{n=0}^{\infty}\left|b_{n}\right| r^{n} \leq & +\frac{1-a^{2}}{1+\gamma} \frac{r}{1-r}+\left(\frac{1}{1+a}+\frac{r}{1-r}\right)\left(\frac{1-a^{2}}{1+\gamma}\right)^{2} \frac{r^{2}}{1-r^{2}} \\
& -\left(\frac{1}{1+\left|b_{0}\right|}+\frac{r}{1+r}\right) \sum_{n=1}^{\infty}\left|b_{n}\right|^{2} r^{2 n}
\end{aligned}
$$

Since

$$
\sum_{n=1}^{\infty}\left|a_{n}\right| r^{n}=\sum_{n=0}^{\infty}\left|b_{n}\right| r^{n+1}=r \sum_{n=0}^{\infty}\left|b_{n}\right| r^{n},
$$

in view of (3.20) and (3.21), we obtain

$$
\begin{aligned}
\sum_{n=1}^{\infty}\left|a_{n}\right| r^{n} \leq & r\left(a+\frac{1-a^{2}}{1+\gamma} \frac{r}{1-r}\right)+\left(\frac{1}{1+a}+\frac{r}{1-r}\right)\left(\frac{1-a^{2}}{1+\gamma}\right)^{2} \frac{r^{3}}{1-r^{2}} \\
& -\left(\frac{1}{1+a}+\frac{r}{1-r}\right) \sum_{n=1}^{\infty}\left|a_{n+1}\right|^{2} r^{2 n+1} \\
= & r a+\left(\frac{1-a^{2}}{1+\gamma}\right) \frac{r^{2}}{1-r}+\left(\frac{1}{1+a}+\frac{r}{1-r}\right)\left(\frac{1-a^{2}}{1+\gamma}\right)^{2} \frac{r^{3}}{1-r^{2}} \\
& -\left(\frac{1}{1+a}+\frac{r}{1-r}\right) \sum_{n=2}^{\infty}\left|a_{n}\right|^{2} r^{2 n-1} .
\end{aligned}
$$


Further simplification shows that

$$
\begin{aligned}
& \sum_{n=1}^{\infty}\left|a_{n}\right| r^{n}+\left(\frac{1}{1+a}+\frac{r}{1-r}\right) \sum_{n=2}^{\infty}\left|a_{n}\right|^{2} r^{2 n-1} \\
& \leq r a+\left(\frac{1-a^{2}}{1+\gamma}\right) \frac{r^{2}}{1-r}+\left(\frac{1}{1+a}+\frac{r}{1-r}\right)\left(\frac{1-a^{2}}{1+\gamma}\right)^{2} \frac{r^{3}}{1-r^{2}} \\
& :=\mathcal{T}(a) .
\end{aligned}
$$

It is easy to see that $\mathcal{T}$ can be represented as

$$
\mathcal{T}(a)=a r+A\left(1-a^{2}\right)+B(1-a)\left(1-a^{2}\right)+C\left(1-a^{2}\right)^{2},
$$

where

$$
\begin{aligned}
& A=A(r)=\frac{r^{2}}{(1+\gamma)(1-r)}, \\
& B=B(r)=\frac{r^{3}}{(1+\gamma)^{2}\left(1-r^{2}\right)} \quad \text { and } \\
& C=C(r)=\frac{r^{4}}{(1+\gamma)^{2}(1-r)\left(1-r^{2}\right)} .
\end{aligned}
$$

Clearly, $B$ and $C$ are positive. We note that,

$$
\begin{aligned}
\mathcal{T}^{\prime}(a) & =r-2 A a+B\left(3 a^{2}-2 a-1\right)+4 C\left(a^{3}-a\right), \\
\mathcal{T}^{\prime \prime}(a) & =-2 A+2 B(3 a-1)+4 C\left(3 a^{2}-1\right) \text { and } \\
\mathcal{T}^{\prime \prime \prime}(a) & =6 B+24 C a .
\end{aligned}
$$

Since $B$ and $C$ are positive, it follows that $\mathcal{T}^{\prime \prime \prime}(a)>0$ for $a \in[0,1]$. In other words, $\mathcal{T}^{\prime \prime}$ is an increasing function of $a$ in $[0,1]$. Therefore,

$$
\mathcal{T}^{\prime \prime}(a) \leq \mathcal{T}^{\prime \prime}(1)=-2 A+4 B+8 C=\frac{2 r^{2}}{(1+\gamma)^{2}(1-r)\left(1-r^{2}\right)} L(r)
$$

where

$$
L(r)=4 r^{2}+2 r(1-r)-(1+\gamma)\left(1-r^{2}\right)=(1+r)(r(3+\gamma)-(1+\gamma)) .
$$

It is easy to see that $L(r) \leq 0$ for $r \leq r_{0}=(1+\gamma) /(3+\gamma)$. Hence, $\mathcal{T}^{\prime \prime}(a) \leq 0$ for $a \in[0,1]$ which implies that $\mathcal{T}^{\prime}$ is decreasing in $[0,1]$. Therefore, for $r \leq r_{0}=$ $(1+\gamma) /(3+\gamma)$, we obtain

$$
\mathcal{T}^{\prime}(a)>\mathcal{T}^{\prime}(1)=1-2 A=r \frac{1+\gamma-r(3+\gamma)}{(1+\gamma)(1-r)}
$$

Clearly, for $r \leq r_{0}$, we have $\mathcal{T}^{\prime}(1) \geq 0$ for all $a \in[0,1]$. Since $\mathcal{T}^{\prime}(a) \geq 0$ in [0,1], $\mathcal{T}$ is an increasing function in $[0,1]$, and hence, we have $\mathcal{T}(a) \leq \mathcal{T}(1)=r$. A simple computation shows that

$$
\sum_{n=1}^{\infty}\left|a_{n}\right| r^{n}+\left(\frac{1}{1+\left|a_{1}\right|}+\frac{r}{1-r}\right) \sum_{n=2}^{\infty}\left|a_{n}\right|^{2} r^{2(n-1)} \leq 1 \quad \text { for } r \leq r_{0}=\frac{1+\gamma}{3+\gamma}
$$

To show that the sharpness of the radius we consider the function $f_{a}^{*}$ by

$$
f_{a}^{*}(z):=z f_{a}(z)=z\left(\frac{a-\gamma-(1-\gamma) z}{1-a \gamma-a(1-\gamma) z}\right)=C_{0} z-\sum_{n=1}^{\infty} C_{n} z^{n+1} \quad \text { for } \quad z \in \mathbb{D},
$$


where

$$
C_{0}=\frac{a-\gamma}{1-a \gamma} \quad \text { and } \quad C_{n}=\frac{\left(1-a^{2}\right)}{a(1-a \gamma)}\left(\frac{a(1-\gamma)}{1-a \gamma}\right)^{n}
$$

For $n \geq 2$, it is easy to see that $a_{1}\left(f_{a}\right)=C_{0}$ and $a_{n}\left(f_{a}\right)=-C_{n-1}$. For $\gamma \in[0,1]$, and $a>\gamma$, a simple calculation shows that

$$
\begin{aligned}
D(r):= & \sum_{n=1}^{\infty}\left|C_{n}\right| r^{n}+\left(\frac{1}{1+\left|C_{0}\right|}+\frac{r}{1-r}\right) \sum_{n=2}^{\infty}\left|C_{n}\right|^{2} r^{2 n-1} \\
= & \left(\frac{a-\gamma}{1-a \gamma}\right) r+\sum_{n=2}^{\infty} \frac{\left(1-a^{2}\right)}{a(1-a \gamma)}\left(\frac{a(1-\gamma)}{1-a \gamma}\right)^{n-1} r^{n} \\
& +\left(\frac{1}{1+\left|C_{0}\right|}+\frac{r}{1-r}\right) \sum_{n=2}^{\infty} \frac{\left(1-a^{2}\right)^{2}}{a^{2}(1-a \gamma)^{2}}\left(\frac{a(1-\gamma)}{1-a \gamma}\right)^{2(n-1)} r^{2 n-1} \\
= & \left(1-\frac{1-a}{1-a \gamma} \chi(r)\right) r
\end{aligned}
$$

where

$$
\begin{aligned}
\chi(r):= & +\gamma-\frac{(1+a)(1-\gamma) r}{1-a \gamma-a(1-\gamma) r} \\
& -\left(\frac{1-a \gamma}{(1+a)(1-\gamma)}+\frac{r}{1-r}\right) \frac{(1+a)\left(1-a^{2}\right)}{1-a \gamma} \frac{(1-\gamma)^{2} r^{2}}{(1-a \gamma)^{2}-a^{2}(1-\gamma)^{2} r^{2}} .
\end{aligned}
$$

Note that

$$
\begin{aligned}
\chi^{\prime}(r)= & -\frac{(1+a)(1-\gamma)(1-a \gamma)}{(1-a \gamma-a(1-\gamma) r)^{2}}-\frac{(1+a)\left(1-a^{2}\right)}{(1-a \gamma)(1-r)^{2}} \frac{(1-\gamma)^{2} r^{2}}{(1-a \gamma)^{2}-a^{2}(1-\gamma)^{2} r^{2}} \\
& -\left(\frac{1-a \gamma}{(1+a)(1-\gamma)}+\frac{r}{1-r}\right) \frac{2(1-a \gamma)(1+a)\left(1-a^{2}\right)(1-\gamma)^{2} r}{\left((1-a \gamma)^{2}-a^{2}(1-\gamma)^{2} r^{2}\right)^{2}}<0 .
\end{aligned}
$$

Therefore, $\chi$ is strictly decreasing function in $r \in(0,1)$. Hence, for $r>r_{0}$, we have $\chi(r)<\chi\left(x_{0}\right)$. It is worth to point out that

$$
\lim _{a \rightarrow 1} \chi\left(r_{0}\right)=1+\gamma-\frac{2(1-\gamma) r_{0}}{1-\gamma-(1-\gamma) r_{0}}=1+\gamma-\frac{2 r_{0}}{1-r_{0}}=0
$$

This shows that $\chi(r) \leq 0$ for $r>r_{0}$ as $a \rightarrow 1$, and hence $D(r)>r$ for $r>r_{0}$. Therefore,

$$
\sum_{n=1}^{\infty}\left|a_{n}\right| r^{n}+\left(\frac{1}{1+\left|a_{1}\right|}+\frac{r}{1-r}\right) \sum_{n=2}^{\infty}\left|a_{n}\right|^{2} r^{2(n-1)}>1
$$

and hence $r_{0}$ is the best possible. This completes the proof.

Acknowledgment. The authors thank the referee for valuable suggestions which greatly helped to improve the clarity of the exposition in this paper. The first author is supported by the Institute Post Doctoral Fellowship of IIT Bhubaneswar, India, the second author is supported by SERB-MATRICS, and third author is supported by CSIR, India. 


\section{References}

[1] Abu-Muhanna, Y.: Bohr's phenomenon in subordination and bounded harmonic classes. Complex Var. Elliptic Equ. 55, 2010, 1071-1078.

[2] Abu-Muhanna, Y., and R. M. Ali: Bohr's phenomenon for analytic functions into the exterior of a compact convex body. - J. Math. Anal. Appl. 379, 2011, 512-517.

[3] Abu Muhanna, Y., and R. M. Ali: Bohr's phenomenon for analytic functions and the hyperbolic metric. - Math. Nachr. 286, 2013, 1059-1065.

[4] Abu Muhanna, Y., R. M. Ali, Z. C. NG, and S. F. M. Hasni: Bohr radius for subordinating families of analytic functions and bounded harmonic mappings. - J. Math. Anal. Appl. 420, 2014, 124-136.

[5] Abu Muhanna, Y., R. M. Ali, and S. Ponnusamy: On the Bohr inequality. - In: Progress in Aporoximation Theory and Applicable Complex Analysis (edited by N.K. Govil et al.), Springer Optim. Appl. 117, 2016, 265-295.

[6] Aizenberg, L.: Multidimensional analogues of Bohr's theorem on power series. - Proc. Amer. Math. Soc. 128, 2000, 1147-1155.

[7] Aizenberg, L.: Generalization of results about the Bohr radius for power series. - Stud. Math. 180, 2007, 161-168.

[8] Aizenberg, L., A. Aytuna, and P. Djakov: Generalization of theorem on Bohr for bases in spaces of holomorphic functions of several complex variables. - J. Math. Anal. Appl. 258, 2001, 429-447.

[9] Ali, R. M., and Z.C. NG: The Bohr inequality in the hyperbolic plane. - Complex Var. Elliptic Equ. 63:11, 2018, 1539-1557.

[10] Ali, R. M., Z. Abdulhadi, and Z. C. NG: The Bohr radius for starlike logharmonic mappings. - Complex Var. Elliptic Equ. 61:1, 2016, 1-14.

[11] Ali, R. M., R. W. Barnard, and A. Yu. Solynin: A note on Bohr's phenomenon for power series. - J. Math. Anal. Appl. 449, 2017, 154-167.

[12] Ali, R. M., N. K. Jain, and V. Ravichandran: Bohr radius for classes of analytic functions. - Results Math. 74, 2019, 179.

[13] Alkhaleefah, S. A., I. R. Kayumov, and S. Ponnusamy: On the Bohr inequality with a fixed zero coefficient. - Proc. Amer. Math. Soc. 147, 2019, 5263-5274.

[14] Alkhaleefah, S. A., I. R. Kayumov, and S. Ponnusamy: Bohr-Rogosinski inequalities for bounded analytic functions. - Lobachevskii J. Math. (to appear).

[15] Allu, V., and H. Halder: Bohr radius for certain classes of starlike and convex univalent functions - J. Math. Anal. Appl. 493:1, 2021, 124519.

[16] Allu, V., and H. Halder: Bohr phenomenon for certain subclasses of harmonic mappings. - Bull. Sci. Math. 173, 2021, 103053.

[17] Allu, V., and H. Halder: Bohr phenomenon for certain close-to-convex analytic functions. - Comput. Methods Funct. Theory, 2021.

[18] Allu, V., and H. Halder: Bohr inequality for certain harmonic mappings. arXiv:2009.08683.

[19] Aytuna, A., and P. DJakov: Bohr property of bases in the space of entire functions and its generalizations. - Bull. London Math. Soc. 45:2, 2013, 411-420.

[20] Bénéteau, C., A. Dahlner, and D. Khavinson: Remarks on the Bohr phenomenon. Comput. Methods Funct. Theory 4:1, 2004, 1-19.

[21] Bhowmik, B., and N. DAs: Bohr phenomenon for operator valued functions with fixed initial coefficients. - arXiv:2003.05810.

[22] Bноwмiк, B., and N. DAs: On the Bohr phenomenon for complex valued and vector valued functions. - arXiv:2011.12766. 
[23] BoAs, H. P., and D. Khavinson: Bohr's power series theorem in several variables. - Proc. Amer. Math. Soc. 125, 1997, 2975-2979.

[24] Bohr, H.: A theorem concerning power series. - Proc. Lond. Math. Soc. s2-13, 1914, 1-5.

[25] Dixon, P. G.: Banach algebras satisfying the non-unital von Neumann inequality. - Bull. London Math. Soc. 27:4, 1995, 359-362.

[26] Evdoridis, S., S. Ponnusamy, and A. Rasila: Improved Bohr's inequality for locally univalent harmonic mappings. - Indag. Math. (N.S.) 30:1, 2019, 201-213.

[27] Evdoridis, S., S. Ponnusamy, and A. Rasila: Improved Bohr's inequality for shifted disks. - Results Math. 76:14, 2021, 15 pp.

[28] Fournier, R., and St. Ruscheweyh: On the Bohr radius for simply connected domains. CRM Proc. Lecture Notes 51, 2010, 165-171.

[29] Huang, Y., M.-S. Liu, and S. Ponnusamy: Refined Bohr-type inequalities with area measure for bounded analytic functions. - Anal. Math. Phys. 10:50, 2020.

[30] Ismagilov, A., I. R. Kayumov, and S. Ponnusamy: Sharp Bohr type inequality. - J. Math. Anal. Appl. 489, 2020, 124147.

[31] Kayumov, I. R., and S. Ponnusamy: Bohr's inequalities for the analytic functions with lacunary series and harmonic functions. - J. Math. Anal. Appl. 465, 2018, 857-871.

[32] Kayumov, I. R., and S. Ponnusamy: On a powered Bohr inequality. - Ann. Acad. Sci. Fenn. Math. 44: 2019, 301-310.

[33] Kayumov, I. R., and S. Ponnusamy: Improved version of Bohr's inequalities. - C. R. Math. Acad. Sci. Paris 358:5, 2020, 615-620.

[34] Kayumov, I. R., and S. Ponnusamy: Bohr-Rogosinski radius for analytic functions. arXiv: 1708.05585.

[35] Kayumov, I. R., S. Ponnusamy, and N. Shakirov: Bohr radius for locally univalent harmonic mappings. - Math. Nachr. 291, 2018, 1757-1768.

[36] Liu, G., Z. Liu, and S. Ponnusamy: Refined Bohr inequality for bounded analytic functions. - Bull. Sci. Math. 173, 2021, 103054.

[37] Liu, M. S., and S. Ponnusamy: Multidimensional analogues of refined Bohr's inequality. Proc. Amer. Math. Soc. 149, 2021, 2133-2146.

[38] Ponnusamy, S., and K.-J. Wirths: Bohr type inequalities for functions with a multiple zero at the origin. - arXiv.2006.06441.

[39] Rogosinski, W.: Uber Bildschranken bei Potenzreihen und ihren Abschnitten. - Math. Z. 17, 1923, 260-276.

[40] Ruscheweyh, St.: Two remarks on bounded analytic functions. - Serdica 11:1, 1985, 731-732.

[41] Sidon, S.: Uber einen satz von Hernn Bohr. - Math. Z. 26, 1927, 731-732.

[42] Tomic, M.: Sur un theoreme de H. Bohr. - Math. Scand. 11, 1962, 103-106.

Received 30 November 2020 • Accepted 5 March 2021 • Published online 2 December 2021

Molla Basir Ahamed

Indian Institute of Technology Bhubaneswar

School of Basic Science

Bhubaneswar-752050, Odisha, India

mba15@iitbbs.ac.in
Vasudevarao Allu

Indian Institute of Technology Bhubaneswar

School of Basic Science

Bhubaneswar-752050, Odisha, India

avrao@iitbbs.ac.in 
Himadri Halder

Indian Institute of Technology Bhubaneswar

School of Basic Science

Bhubaneswar-752050, Odisha, India

hh11@iitbbs.ac.in 